\title{
A H3K9me2-Binding Protein AGDP3 Limits DNA Methylation and Transcriptional Gene Silencing in Arabidopsis
}

Xuelin Zhou ${ }^{1,3, *}$, Mengwei Wei ${ }^{1,3, *}$, Wenfeng Nie ${ }^{1}$, Yue Xi ${ }^{1,3}$, Xuan Du ${ }^{2}$, Li Peng ${ }^{1}$, Qijie

Zheng ${ }^{1}$, Kai Tang ${ }^{1,4}$, Viswanathan Satheesh ${ }^{1}$, Yuhua Wang ${ }^{1}$, Jinyan Luo ${ }^{1}$, Rui Liu ${ }^{2}$, Zhenlin Yang ${ }^{2}$, Yingli Zhong ${ }^{1}$, Guo-Yong $\mathrm{An}^{5}$, Jian-Kang $\mathrm{Zhu}^{1,4}$, Jiamu $\mathrm{Du}^{2, \dagger}$ and Mingguang Lei ${ }^{1,5, \dagger}$

${ }^{1}$ Shanghai Center for Plant Stress Biology, CAS Center for Excellence in Molecular Plant Sciences, Chinese Academy of Sciences, Shanghai 201602, China.

${ }^{2}$ Key Laboratory of Molecular Design for Plant Cell Factory of Guangdong Higher Education Institutes, Institute of Plant and Food Science, School of Life Sciences, Southern University of Science and Technology, Shenzhen 518055, China.

${ }^{3}$ University of Chinese Academy of Sciences, Beijing 100049, China.

${ }^{4}$ Department of Horticulture \& Landscape Architecture, Purdue University, West Lafayette, IN 47906, USA.

${ }^{5}$ State Key Laboratory of Crop Stress Adaptation and Improvement, School of Life Sciences, Henan University, Kaifeng 475004, China.

${ }^{*}$ Co-first authors.

${ }^{\dagger}$ Co-corresponding authors: Jiamu Du (dujm@sustech.edu.cn), Mingguang Lei (mglei@,cemps.ac.cn)

Keywords: AGDP3, ROS1, DNA demethylation, H3K9me2, Epigenetics. 
bioRxiv preprint doi: https://doi.org/10.1101/2021.03.08.434320; this version posted March 8, 2021. The copyright holder for this preprint (which was not certified by peer review) is the author/funder. All rights reserved. No reuse allowed without permission. 


\begin{abstract}
DNA methylation is critical for tuning gene expression to prevent potentially deleterious gene-silencing. The Arabidopsis DNA glycosylase/lyase REPRESSOR OF SILENCING 1 (ROS1) initiates active DNA demethylation and is required for the prevention of DNA hypermethylation at thousands of genomic loci. However, the mechanism recruiting ROS1 to specific loci is not well understood. Here, we report the discovery of Arabidopsis AGENET Domain Containing Protein 3 (AGDP3) as a cellular factor required for ROS1mediated DNA demethylation, and targets ROS1 to specific loci. We found that AGDP3 could bind to the H3K9me2 mark by its AGD12 cassette. The crystal structure of the AGDP3 AGD12 in complex with an H3K9me2 peptide reveals the molecular basis for the specific recognition, that the dimethylated $\mathrm{H} 3 \mathrm{~K} 9$ and unmodified $\mathrm{H} 3 \mathrm{~K} 4$ are specifically anchored into two different surface pockets. Interestingly, a histidine residue located in the methylysine binding aromatic cage enables AGDP3 pH-dependent H3K9me2 binding capacity. Considering the intracellular $\mathrm{pH}$ correlates with the histone acetylation status, our results provide the molecular mechanism for the regulation of ROS1 DNA demethylase by the gene silencing $\mathrm{H} 3 \mathrm{~K} 9 \mathrm{me} 2$ mark and the potential crosstalk with active histone acetylation mark.
\end{abstract}




\section{Introduction}

DNA methylation, characterized by adding a methyl group onto the fifth position of the cytosine, has profound impact on biological processes such as gene regulation, transposable element silencing and genome stability (Robertson, 2005; Slotkin and Martienssen, 2007; Zhang et al., 2018b). In plants, DNA is methylated at specific loci by DNA methyltransferases controlled by the RNA-directed DNA methylation (RdDM) pathway (Law and and Jacobsen 2010; Matzke and Mosher, 2014). Once established, different mechanisms are required to maintain this epigenetic mark, depending on the cytosine context. While the methylation in the symmetric $\mathrm{CG}$ and $\mathrm{CHG}$ (where $\mathrm{H}$ is $\mathrm{C}, \mathrm{A}$ or T) context is copied during DNA replication by DNA methyltransferase 1 (MET1) and chromomethylase $\mathrm{CMT} 3$, respectively, the asymmetrical $\mathrm{CHH}$ methylation is de novo methylated by domain rearranged methyltransferase 2 (DRM2) through RdDM pathway and by CMT2 (Lindroth et al., 2001; Matzke et al. 2009; Zemach et al., 2013). On the other hand, DNA methylation can be actively erased by a class of bifunctional DNA glycosylases/lyases, including REPRESSOR OF SILENCING 1 (ROS1), DEMETER (DME), DME-LIKE 2 (DML2), and DML3 (Zhu, 2009). While DME is preferentially expressed in companion cells of gametes and functions in genomic imprinting (Gehring et al., 2006; Hsieh et al., 2009; Huh et al., 2008; Penterman et al., 2007), DML2, DML3, and ROS1 mainly function in locus-specific DNA demethylation and in preventing transcriptional silencing of endogenous and transgenic loci (Gong et al., 2002; Penterman et al., 2007; Qian et al., 2012).

ROS1 is targeted to specific genomic loci through the Increased DNA Methylation (IDM) complex comprising IDM1, IDM2, IDM3, methyl CpG-binding protein 7 (MBD7) and 
Harbinger transposon-derived proteins HDP1 and HDP2 (Qian et al., 2012; Qian et al., 2014; Lang et al., 2015; Duan et al., 2017). MBD7 preferentially binds to densely methylated CG regions and recruits IDM1 to acetylate histone H3K18 and H3K23 to facilitates ROS1-mediated DNA demethylation (Qian et al., 2012; Lang et al., 2015). At a subset of H2A.Z-enriched loci, MBD9 and SNX1 recognize these acetylated histone and recruit SWR1 complex to deposit H2A.Z, which tethers ROS1 to demethylate DNA methylation (Nie et al., 2019; Sijacic et al., 2019). Recently, it was reported that another methyl DNA binding protein RMB1 can interact with ROS1 to regulate DNA methylation at several loci, independently of the IDM protein complex (Liu et al., 2020).

DNA is compacted around the histone octamer, which can also be covalently modified at the N-terminal tails. These post-translational modifications can change chromatin states and/or recruit some histone readers, which may further modify the histone and even the DNA. For example, $\mathrm{H} 3 \mathrm{~K} 9 \mathrm{me} 2$, a mark rich in pericentromeric heterochromatin regions, is associated with transcriptional repression. It is created by H3K9 methyltransferases KRYPTONITE, SUVH5 and SUVH6, which are recruited mainly by binding CHG methylation. On the other hand, CMT2 and CMT3 can recognize H3K9me2 and are recruited to methylate $\mathrm{CHH}$ and $\mathrm{CHG}$, respectively. Thus, $\mathrm{H} 3 \mathrm{~K} 9$ methylation and DNA methylation form a feedback loop to reinforce the repression (Du et al., 2015). AGENET domain (AGD) belongs to the 'Royal Family' histone readers (Maurer-Stroh et al., 2003). In human, the tandem AGDs of FMRP is able to specifically recognize the H3K79me2 mark (Alpatov et al., 2014; Ramos et al., 2006). In plants, the tandem AGDs of AGENET DOMAIN CONTAINING PROTEIN 1 (AGDP1, also known as ADCP1) can specifically recognize the H3K9me2 mark in vitro and in vivo (Zhang et al., 2018a; Zhao et al., 2019). 
AGDP1 is concentrated in centromeric and pericentromeric regions and recruits some unknown factors to regulate non-CG methylation and transcriptional gene silencing (Zhang et al., 2018; Zhao et al., 2019). However, H3K9me2 methylation is also present in facultative heterochromatin, where the expression of resident genes or nearby genes are dynamically regulated through the life cycle or in response to environmental stimuli. We, therefore, opine that there exists some factor(s) that recognize this repressive mark and then change the modification or recruit some other regulator(s) to prevent transcriptional silencing.

Here, we identified an anti-silencing factor AGENET DOMAIN CONTAINING PROTEIN 3 (AGDP3) with two pairs of tandem AGDs. We found that AGDP3 prevents transgenes and some endogenous loci from hypermethylation. We further found that AGDP3 can specifically recognize the gene silencing H3K9me2 mark by its first tandem AGD cassette. Our structural studies revealed the molecular mechanism of the specific recognition of $\mathrm{H} 3 \mathrm{~K} 9 \mathrm{me} 2$ by AGD12 of AGDP3 and further identified AGDP3 as a pHdependent histone reader with lower binding in low $\mathrm{pH}$. The intracellular $\mathrm{pH}$ is correlated with the histone acetylation status. Therefore, our studies revealed the molecular basis for the specific targeting of ROS1 anti-silencing machinery to its substrate methylated DNA loci, which is enriched with $\mathrm{H} 3 \mathrm{~K} 9 \mathrm{me} 2$ and further shed light on the mechanism underlying the crosstalk among DNA methylation, H3K9me2 and histone acetylation.

\section{Results}

\section{AGDP3 prevents gene silencing}


To identify potential anti-silencing factors in Arabidopsis, we performed a forward preestablished genetic screen with transgenic plants expressing the cauliflower mosaic virus 35S promoter-driven sucrose transporter 2 (35S::SUC2). Overexpression of SUC2 results in sucrose over-accumulation and seedlings have short roots when grown on medium containing 1\% sucrose compared with Col-0 wild-type plants (Lei et al., 2014). We isolated two mutant alleles on sucrose-containing media, agdp3-1 and agdp3-2, displaying longroot phenotype compared with $35 S:: S U C 2$ (hereafter referred to as WT) plants (Fig. 1a). Consistent with the root phenotype, the $S U C 2$ gene expression was silenced in agdp3-1 mutant (Fig. 1b). The WT also expressed hygromycin phosphotransferase II (HPTII) gene driven by double $35 S(2 \times 35 S)$ promoter and conferred hygromycin resistance (Lei et al., 2014). This transgene was also silenced in agdp3-1 and agdp3-2 mutants, showing hygromycin sensitive phenotype (Fig. 1a and 1c). Then, we compared the transcriptomes, and found that 51 endogenous genes were significantly downregulated in agdp3-1 mutant when compared with the WT $(\mathrm{FDR}=0.05, \mathrm{FC}>2)$ (Fig. 1d). GO enrichment analysis indicated that AGDP3 mutation may affect some biological processes (Fig. 1d). These results show that AGDP3 is required for preventing silencing of both transgenes and some endogenous genes.

\section{AGDP3 regulates DNA demethylation}

Through map-based cloning, we found that agdp3-1 has $\mathrm{G}$ to A mutation at the end of the second intron of At2G47230 causing a splicing defect. Also, a G to A mutation in the first exon changes Asp92 to Asn in agdp3-2 (Fig. 1e). To confirm that the agdp3 mutations were responsible for the silencing of $35 S:: S U C 2$, the expression of $A G D P 3$ fused with a 
$3 x F L A G$ tag driven by its native promoter ( $p A G D P 3: \because A G D P 3-3 x F L A G)$ in agdp3-1 restored 35S::SUC2 gene expression and rescued the short root phenotype (Fig. 1f and 1g).

AGDP3 was predicted to contain an N-terminal AGENET domain (AGD) with four tandem AGD motifs and a C-terminal plant-specific DUF724 domain with unknown function. The AGENET domain-contain protein AGDP1 was reported to be required for transcriptional gene silencing and DNA methylation (Zhang et al., 2018). To determine whether transgene silencing in the agdp3-1 and agdp3-2 mutants is also associated with DNA methylation, we examined the DNA methylation levels of the $35 S$ promoter. Bisulfite sequencing showed that $35 \mathrm{~S}$ promoter was hypermethylated, especially in region $\mathrm{A}$, in agdp3-1 mutant (Supplemental Fig. 1a). To support the hypothesis that the transgene silencing was caused by increased DNA methylation, we treated the seedlings with the DNA methylation inhibitor 5-aza-2'-deoxycytidine. Not only the root phenotype, but the transgene silencing was also rescued in agdp3-1 and agdp3-2 mutants (Supplemental Fig. $1 \mathrm{~b}$ and $1 \mathrm{c})$.

To investigate whether AGDP3 also prevents endogenous loci from hypermethylation, we firstly used Chop PCR to determine DNA methylation level at the 3' region of AT1G26400 and the promoter of AT4G18650, two genomic loci hypermethylated in ros 1 mutant plant (Qian et al., 2012). Both loci were also hypermethylated in agdp3-1 plants (Supplemental Fig. 1d). The T-DNA insertion mutant allele agdp3-3 in Col-0 background without 35S::SUC2 transgene also showed hypermethylation at both loci (Supplemental Fig. 1d). To understand the extent of agdp3 mutation on genome-wide DNA methylation, we compared the methylomes of agdp3-3 and wild-type Col-0 plants. In general, the overall DNA methylation patterns in genes and transposons were not altered by agdp3 mutation. 
However, we identified 1475 differentially methylated regions (DMRs) in the agdp3-3 mutant plants compared to WT plants (Fig. 2a). The DMRs are distributed across each chromosome and about $32 \%, 39 \%$ and $27 \%$ of them are in the genic, TE and intergenic regions, respectively (Supplemental Fig. 2a and 2b). Among the 909 hyper-DMRs with significantly increased DNA methylation in agdp3-3, about 51\% and 54\% are also hypermethylated in ros 1 and $r d d$ mutants, respectively. However, only $22 \%$ of the hyperDMRs in agdp3-3 overlap with those in $i d m 1-1$ mutant (Fig. 2a and 2b). Together, these results indicate that AGDP3 regulates active DNA demethylation whole-genome wide in an IDM1-independent way.

\section{AGDP3 associates with genomic region of H3K9me2 mark}

AGDP3 has an Agenet domain, suggesting that it may act as a histone reader to recognize some specific histone modification. The Agenet domains are plant-specific histone readers that recognize different histone modifications. To identify specific targets of AGDP3, we performed Cleavage Under Targets and Tagmentation (CUT\&Tag) assay, using agdp3-3 mutant transformed with $p A G D P 3:: A G D P 3-3 x F L A G$, which complemented the DNA hypermethylation phenotype. We identified 252 peaks of AGDP3 enrichment on chromatin, among which 218 (86.5\%) overlapped with H3K9me2 peaks (Fig. 3). This result suggests that AGDP3 may recognize some specific loci with H3K9me2 mark and regulate DNA demethylation.

\section{AGDP3 AGD12 binds to H3K9me2 but AGD34 does not}

The Arabidopsis thaliana AGDP3 (AtAGDP3) possesses four tandem AGDs (AGD1-4) at its N-terminus, which can be divided into two cassettes (AGD12 and AGD34) and a C- 
terminal Domain of Unknown Function 724 (DUF724) domain (Fig. 4a) (Marchler-Bauer et al., 2017). In plants, the tandem AGD cassette has been recently reported to function as a histone mark reader module to recognize the H3K9me2 mark (Chen, 2019; Harris and Jacobsen, 2019; Zhang et al., 2018a; Zhao et al., 2019). To explore the potential histone mark binding property of AGDP3, we expressed the AGD1-4 of AtAGDP3 and performed ITC-based in vitro binding assay (Supplemental Fig. 3a). The AGD1-4 of AtAGDP3 clearly shows a binding preference towards the H3K9me2 mark over other common histone marks (Supplemental Fig. 3a and 3b). The binding yields a binding ratio near 1 (Supplemental Fig. 3b), indicating that only one pair of tandem AGD cassettes is responsible for the H3K9me2 binding. To check which tandem AGDs recognize H3K9me2, we split the AGD1-4 to AGD12 and AGD34. However, both the isolated AGD12 and AGD34 of AtAGDP3 aggregated during purification and could not be used for further binding assay. Further, we chose its orthologs from other species for testing. We used the Nicotiana tabacum AGDP3 (NtAGDP3), which possesses the same domain architecture and shares 38\% and 29\% sequence identities with the AtAGDP3 AGD1-4 and DUF724 (Fig. 4a and Supplemental Fig. 4), respectively, for further experimentation. The NtAGDP3 AGD1-4 selectively binds to methylated H3K9 mark with a preference for H3K9me2, too (Fig. 4b and 4c). The AGD12 of NtAGDP3, but not AGD34, can specifically recognize the methylated $\mathrm{H} 3 \mathrm{~K} 9$ with a preference on the dimethylation, which is similar to the AtAGDP3 (Fig. 4d).

\section{Overall structure of AGD1-4}

To investigate the molecular details of the recognition, we further performed structural studies. We determined the crystal structure of AtAGDP3 AGD1-4 and the structure was 
refined to $2.6 \AA$ resolution (Fig. 4e and Table S1). The structure is composed of two tandem AGDs with AGD1 and AGD4 flanking on the two sides and AGD2 and AGD3 in the middle (Fig. 4E). Overall, both the AGD12 and AGD34 adopt classical tandem AGD folds resembling previously reported plant AGDP1 and human FMRP tandem AGDs (Myrick et al., 2015; Zhang et al., 2018a; Zhao et al., 2019b). The superimposition of AGDP3 AGD12 and AGD34 yields an RMSD of $2.0 \AA$ for 127 aligned Cas, indicating quite similar overall structures. The interface between AGD2 and AGD3 is composed by a hydrophobic core and two pairs of salt bridge interactions on the sides (Fig. 4f).

\section{Structure basis for the recognition of H3K9me2 by AGD12 of AGDP3}

To further investigate the molecular basis for the recognition of H3K9me2 by AGD12 of AGDP3, we tried to obtain the complex structure between AGDP3 and an H3K9me2 peptide. After extensive testing, we successfully got the crystal structure of NtAGDP3 AGD12 in complex with an $\mathrm{H} 3(1-15) \mathrm{K} 9 \mathrm{me} 2$ peptide at $2.3 \AA$ resolution (Fig. 5a and Table S1). The NtAGDP3 AGD12 resembles the AtAGDP3 AGD12 with an RMSD of $1.6 \AA$ for 136 Cas upon superimposition (Fig. 5a). Similar to the AGDP1 AGD12-H3K9me2 complex, the peptide features a unique helical conformation that the H3K4 to H3A7 of the peptide forms a short $\alpha$-helix (Fig. 5a). The peptide binds onto a negatively charged continuous surface between the two AGDs with the H3K4me0 and H3K9me2 side chains inserting into two surface pockets on the AGD1 and AGD2, respectively (Fig. 5a and 5b). The interaction between AGDP3 and $\mathrm{H} 3 \mathrm{~K} 9 \mathrm{me} 2$ peptide focuses on three regions. At the N-terminus, the main chain carbonyl of AGD1 residue Ser55 forms two hydrogen bonds with the amino and amide groups of H3A1 and H3R2, respectively (Fig. 5c). The main chain carbonyl and side chain guanidine groups of H3R2 form hydrogen bonding and/or 
salt bridge interactions with Val57 of AGD1 and Asp99 of AGD2, respectively (Fig. 5c).

The unmodified H3K4 forms salt bridge and hydrogen bonding interactions with Glu68 and Tyr53 of AGD1, respectively (Fig. 5d). Most importantly, the dimethyllysine of H3K9me2 is accommodated by an aromatic cage formed by Phe119, Trp102 and His97 (Fig. 5e), resembling other methyllysine readers (Patel, 2016). The mutations of the aromatic cage residues lead to the disruption of the binding between AGDP3 and $\mathrm{H} 3 \mathrm{~K} 9 \mathrm{me}$ 2, which is revealed by our ITC data (Fig. 5f). It is worth noting that all the NtAGDP3 residues involved in the interactions with $\mathrm{H} 3 \mathrm{~K} 9 \mathrm{me} 2$ are strictly conserved in both sequence and structure with AtGADP3 (Fig. 6a) and the binding is similar to AGDP1 (Fig. 6b) (Zhang et al., 2018a; Zhao et al., 2019). However, these H3K9me2 interacting residues are not conserved in the AGD34 of AtAGDP3 structurally (Fig. 6c), consistent with our biochemical data that AGD12 but not AGD34 of AGDP3 recognizes H3K9me2 mark. Meanwhile, the AGD34 may have functions other than the H3K9me2 binding, which remains to be elucidated in the future.

\section{AGDP3 is a $\mathrm{pH}$ sensitive $\mathrm{H3K9me2}$ reader}

Sine the $\mathrm{pKa}$ of the protonated imidazole group of the histidine side chain is around 6.07.5 in folded proteins and the plant cell nucleus has a pH of $7.2 \pm 0.2$ (Shen et al., 2013), the existence of a histone residue in the methyllysine binding aromatic cage raises the possibility that the binding of $\mathrm{H} 3 \mathrm{~K} 9 \mathrm{me} 2$ by AGDP3 is $\mathrm{pH}$ dependent. As the AGD12 of NtAGDP3 precipitates heavily in low $\mathrm{pH}$, we used AtAGDP3 AGD1-4 for testing. Consequently, the protonated histidine containing aromatic cage of AtAGDP3 showed about 5-fold decreased binding towards $\mathrm{H} 3 \mathrm{~K} 9 \mathrm{me} 2$ in $\mathrm{pH} 6.0$ than in $\mathrm{pH} 7.5$ (Fig. $5 \mathrm{~g}$ ). In contrast, the H90Y mutant of AtAGDP3, which corresponds to H97Y of NtAGDP3, loses 
the $\mathrm{pH}$ sensitivity (Fig. 6d), confirming that the histidine residue of the aromatic cage is responsible for the $\mathrm{pH}-$ dependent binding of $\mathrm{H} 3 \mathrm{~K} 9 \mathrm{me} 2$ by AGDP3. This type of $\mathrm{pH}$ dependent binding of histone mark is similar to the recognition of $\mathrm{H} 3 \mathrm{~K} 4 \mathrm{me} 3$ by the Drosophila Protein Partner of Sans-fille (PPS) and the recognition of H3K122suc by Glioma-Amplified Sequence-41 (GAS41) (Tencer et al., 2017; Wang et al., 2018), indicating this might be a common regulatory mechanism for histone readers.

\section{Discussion}

Active DNA demethylation is a dynamic process that is manipulated by many different regulatory factors and enzymes that work coordinately. Even though ROS1 has been extensively studied in enzymology, the mechanisms of recruitment to its target sites are still poorly understood in plants. In this study, we isolated the AGENET domain containing protein AGDP3 as an anti-silencing factor from a forward genetic screen, and find that ADGP3 functions as a cellular DNA demethylation regulator that inhibits DNA hypermethylation at multiple genomic regions. Our results indicate that AGDP3 prevents genome-wide DNA methylation and protects endogenous genes from silencing, showing important roles in epigenetic regulation and active DNA demethylation. Epigenetic modification enzymes are usually associated with DNA- or chromatin-binding proteins or domains for specific targeting (DesJarlais and Tummino, 2016). The current study suggests that ADGP3 may form a protein complex with ROS1 to function in active DNA demethylation. So far, two different active DNA methylation pathways have been identified in Arabidopsis: H2A.Z-dependent recruitment of ROS1 to specific genomic regions binding by IDM1 and MBD7 in the IDM complex (Nie et al., 2019; Qian et al., 
2012) and RWD40-dependent recruitment of ROS1 to specific target loci binding by RMB1 in RWD40 complex (Liu et al., 2020). However, it should be noted that even additive DMRs contributed by these two pathways are much fewer than the hyper-DMRs induced by dysfunction of ROS1, which indicates the complexity of active DNA methylation in plants. The ADPG3 identified in this study contributes to a subset of genomic methylated regions targeted by ROS1, which is different from the IDM complexor RWD40 complex-mediated pathway. Hence, our study enriches the mechanisms of active DNA demethylation in plants.

Recently, the crystal structures of tandem AGDs of AGDP1 in complex with H3K9me2 were reported (Zhang et al., 2018a; Zhao et al., 2019). The superimposition of AGDP1 AGD12-H3K9me2 complex and AGDP3 AGD12-H3K9me2 complex yielded an RMSD of $1.2 \AA$ for 136 Cas, revealing almost identical overall structures (Fig. 6b). Most of the key residues involved in peptide recognition are strictly conserved, and the most obvious difference is the aromatic cage residue His97 in NtAGDP3 which is equivalent to the Tyr122 residue in RsAGDP1 (Fig. 6b). The existence of a histidine residue in the aromatic cage enables AGDP3 pH dependent binding of H3K9me2 (Fig. 5g). As AGDP1 is considered as a plant functional analog of animal HP1 protein and is tightly associated with heterochromatic H3K9me2 and DNA methylation (Zhang et al., 2018a; Zhao et al., 2019), it is convictive for AGDP1 to possess constitutive binding towards the gene silencing mark $\mathrm{H} 3 \mathrm{~K} 9 \mathrm{me} 2$, no matter how $\mathrm{pH}$ changes. In contrast to DNA methylation, DNA demethylation occurs more dynamically as an on-or-off switch and requires the changing of the micro-epigenetic environment from silencing to activation. Especially, the 
AGDP3 associates with H3K9me2 but not the ROS1 associated H3K18ac and H3K27me3 marks, indicating that AGDP3 targeting to chromatin is more dynamically regulated.

It was reported that global histone acetylation, a signal of gene activation, is associated with high intracellular $\mathrm{pH}(\mathrm{pH} 7.4)$, while histone deacetylation is with low intracellular pH (pH 6.5) in animal cells (McBrian et al., 2013). Considering the common relationship between histone modification and metabolism, we think the plant cell may possess a similar phenomenon. Therefore, we can build a plausible molecular model for the action of AGDP3 in ROS1-mediated anti-silencing (Fig. 7). Once the chromatin loci are marked by the gene activation signal of histone acetylation, probably including the H3K18ac, the histone acetylation-associated higher $\mathrm{pH}$ enables AGDP3 to specifically bind to $\mathrm{H} 3 \mathrm{~K} 9 \mathrm{me} 2$ marked chromatin loci and to further recruit ROS1 to mediate DNA demethylation for encountering gene silencing (Fig. 7). In the gene silencing process, the dropping of histone deacetylation-associated $\mathrm{pH}$ may disrupt the binding between AGDP3 and the chromatin, which may further prevent the ROS1 anti-silencing machinery to approach (Fig.7), thereby keeping the DNA methylation mark and maintaining gene silencing chromatic states. Although there is no evidence that the histone acetylation can directly recruit ROS1 anti-silencing complex or not, it can increase the $\mathrm{pH}$ of the microenvironment of chromatin, which further enables H3K9me2 binding by AGDP3. Then, AGDP3 associated ROS1 may subsequently specifically demethylate DNA, removing the gene silencing mark. Overall, we speculate that AGDP3 may function as an indirect histone acetylation sensor via direct sensing of the $\mathrm{pH}$ to further connect ROS1 anti-silencing machinery with its substrate, $\mathrm{H} 3 \mathrm{~K} 9 \mathrm{me} 2 / \mathrm{DNA}$ methylation marked silencing 
chromatin loci. Our work sheds light on the potential internal crosstalk among histone acetylation, H3K9me2 and DNA methylation.

\section{Methods}

\section{Plant materials, mutant screening and map-based cloning}

Wild-type (WT) in this study were described previously (Lei et al., 2011). An EMSmutagenized pool of plants was generated and screened for mutants with a long-root phenotype (Wang et al., 2013). M2 seedlings were grown vertically on $1 / 2$ MS plates with $2 \%$ sucrose and $1 \%$ agar and mutants with long-root phenotype among 7 -day-old seedlings were screened. Genetic mapping and gene cloning was performed as described previously (Lei et al., 2014).

\section{Chop-PCR}

Chop-PCR assays were carried out according to Lei et al (2014).

\section{Whole-genome bisulfite sequencing and data analysis}

Genomic DNA was extracted from 14-day-old seedlings using Dneasy Plant Maxi Kit (Qiagen). Bisulfite conversion, library construction, and deep sequencing were performed by the Novogene Co., Ltd. in Beijing, China. DMRs were identified according to Qian et al. (2012) with some modifications.

\section{Cleavage Under Targets and Tagmentation (CUT\&TAG) Assay}


Experiments were carried out using Hyperactive In-Situ ChIP Library Prep Kit for Illumina (pG-Tn5) (Vazyme Biotech Co.,Ltd). Briefly, 1g flower tissue of pAGDP3AGDP3-3xFLAG/agdp3-3 transgenic plants were collected and directly flash frozen in liquid nitrogen and grinded into fine powder after harvest. Cell nucleus were isolated by filtering through 4 layers of miracloth. Following steps were performed as described in the manufacturer's mannual. The WGS library was sent to the Novogene Co., Ltd. in Beijing, China for Illumina sequencing.

\section{Protein expression and purification}

The gene encoding NtAGDP3 AGD12 (1-150) was cloned into a pSumo vector to fuse an N-terminal hexahistidine plus yeast Sumo tag to the target proteins. The plasmid was transformed into the E. coli strain BL21 (DE3) RIL and cultured in LB medium. The protein expression was induced by adding IPTG to a final concentration of $0.15 \mathrm{mM}$ when the OD600 of cell culture reached 0.6. The recombinant expressed protein was purified using HisTrap column (GE Healthcare). The His-Sumo tag was digested by ulp1 protease and removed by a second HisTrap column. The protein was further purified using a Heparin column (GE Healthcare) and a Superdex G200 column (GE Healthcare). The AtAGDP3 AGD1-4 (1-290), AGD12 (1-143), AGD34 (153-290), NtAGDP3 AGD1-4 (1-296) and AGD34 (150-296) were cloned, expressed and purified using the same protocol. The sitedirected mutagenesis was conducted using a PCR-based method. All the mutant proteins were expressed and purified using the same protocol as the wild type protein. All the peptides were purchased from GL Biochem Ltd (Shanghai).

\section{Crystallization and structure determination}


The crystallization was carried out using vapor diffusion sitting drop method at $20^{\circ} \mathrm{C}$. To facilitate crystallization, a surface entropy reduction method was applied to AtAGDP3 AGD1-4 to generate suitable mutations for crystallization (Goldschmidt et al., 2007). The AtAGDP3 AGD1-4 K261A/K262A/E263A mutant was crystallized in a condition of 0.1 M lithium sulfate, 20\% PEG1000 and 0.1M sodium citrate, pH 5.5. For protein-peptide complex formation, the purified NtAGDP3 AGD12 was mixed with an H3(1-15)K9me2 peptide with a molar ratio of $1: 3$ for 2 hours at $4{ }^{\circ} \mathrm{C}$. The complex crystal was obtained in a condition of $25 \%$ PEG 1500, 0.1 M sodium chloride and $0.1 \mathrm{M}$ bis-Tris propane, $\mathrm{pH}$ 9.0. To resolve the phase by heavy atoms, an AtAGDP3 AGD1-4 crystal was soaked in the reservoir solution supplemented with $5 \mathrm{mM}$ ethylmercurithiosalicylic acid at $20^{\circ} \mathrm{C}$ for $2 \mathrm{~h}$. All the crystals were cryo-protected in the reservoir solution supplemented with $20 \%$ glycerol and then flash cooled into liquid nitrogen. All the diffraction data were collected at beamline BL19U1 of the National Center for Protein Sciences Shanghai (NCPSS) at the Shanghai Synchrotron Radiation Facility (SSRF) and further processed using the program HKL3000 (Otwinowski and Minor, 1997). A summary of the statistics of the data collection is listed in Table S1.

The structure of AtAGDP3 AGD1-4 was determined using single-wavelength anomalous diffraction (SAD) method with the mercury derivate data using the program Phenix (Adams et al., 2010). The structure refinement and model building were applied using the programs Phenix and Coot, respectively (Adams et al., 2010; Emsley et al., 2010). The structure of NtAGDP3 AGD12 in complex with H3K9me2 peptide was determined using molecular replacement method using the program Phenix with the structure of RsAGDP1 AGD12-H3K9me2 complex as search model (Adams et al., 2010; Zhang et al., 
2018a). The NtAGDP3 AGD12-H3K9me2 complex data were anisotropic and were truncated by the Diffraction Anisotropy Server (https://services.mbi.ucla.edu/anisoscale/) (Strong et al., 2006). The structure refinement and model building were performed using the same protocol as AtAGDP3 AGD1-4. A summary of the structure refinement statistics is listed in Table S1. The molecular graphics were generated using the program Pymol (Schrödinger, LLC). The sequence alignments were performed using the program T-coffee and illustrated using the ESPript server (Di Tommaso et al., 2011; Robert and Gouet, 2014).

\section{ITC}

The in vitro binding assays were performed using a Microcal PEAQ ITC instrument (Malvern). The sample proteins were dialyzed into a titration buffer of $100 \mathrm{mM} \mathrm{NaCl}$ and $20 \mathrm{mH}$ HEPES, $\mathrm{pH}$ 7.5. For the $\mathrm{pH}$ dependence test, the low $\mathrm{pH}$ buffer of $100 \mathrm{mM} \mathrm{NaCl}$, $20 \mathrm{mM}$ sodium/potassium phosphate, $\mathrm{pH} 7.0$ or $\mathrm{pH} 8.2$ were used. The peptides were dissolved into the same buffer. The titration experiments were performed at $20{ }^{\circ} \mathrm{C}$ and the data were fit using the program Origin 7.0.

\section{Data availability}

X-ray structures have been deposited in the RCSB Protein Data Bank with the accession codes: XXXX for the AtAGDP3 AGD1-4 and XXXX for NtAGDP3 AGD12 in complex with H3K9me2 peptide.

\section{Author contributions}


X.Z., J.L., X.D., R.L. and Z.Y. performed the structural and biochemical experiments. M.W., W.N., Y.X., V.S., H.L., Y.Z., and Y.Y., performed genetic experiments. K.T., L.P., and Q.Z. did the bioinformatic analysis. J.Z., J.D. and M.L. conceived the study, designed the experiments and wrote the paper.

\section{Competing interests}

The authors declare no competing interests.

\section{ACKNOWLEDGMENTS}

We thank the staffs at beamline BL19U1 of the National Center for Protein Sciences Shanghai (NCPSS) at the Shanghai Synchrotron Radiation Facility (SSRF) for the X-ray data collection. This work was supported by the Chinese Academy of Sciences and National Natural Science Foundation of China (31970580) to M.L., and by National Key R\&D Program (2016YFA0503200), Shenzhen Science and Technology Program (JCYJ20200109110403829 and KQTD20190929173906742) and Key Laboratory of Molecular Design for Plant Cell Factory of Guangdong Higher Education Institutes (2019KSYS006) to J.D.

\section{REFERENCES}

Adams, P.D., Afonine, P.V., Bunkoczi, G., Chen, V.B., Davis, I.W., Echols, N., Headd, J.J., Hung, L.W., Kapral, G.J., Grosse-Kunstleve, R.W., et al. (2010). PHENIX: a 
comprehensive Python-based system for macromolecular structure solution. Acta Crystallogr D Biol Crystallogr 66, 213-221.

Alpatov, R., Lesch, B.J., Nakamoto-Kinoshita, M., Blanco, A., Chen, S., Stutzer, A., Armache, K.J., Simon, M.D., Xu, C., Ali, M., et al. (2014). A chromatin-dependent role of the fragile $\mathrm{X}$ mental retardation protein FMRP in the DNA damage response. Cell 157, 869-881.

Chen, R. (2019). The Long-Sought-After Plant Heterochromatin Protein 1. Mol Plant 12, 14-15.

DesJarlais, R., and Tummino, P.J. (2016). Role of Histone-Modifying Enzymes and Their Complexes in Regulation of Chromatin Biology. Biochemistry 55, 1584-1599.

Di Tommaso, P., Moretti, S., Xenarios, I., Orobitg, M., Montanyola, A., Chang, J.M., Taly, J.F., and Notredame, C. (2011). T-Coffee: a web server for the multiple sequence alignment of protein and RNA sequences using structural information and homology extension. Nucleic Acids Res 39, W13-17.

Du, J., Johnson, L.M., Groth, M., Feng, S., Hale, C.J., Li, S., Vashisht, A.A., Wohlschlegel, J.A., Patel, D.J., and Jacobsen, S.E. (2014). Mechanism of DNA methylation-directed histone methylation by KRYPTONITE. Mol Cell 55, 495-504.

Duan, C.G., Wang, X., Xie, S., Pan, L., Miki, D., Tang, K., Hsu, C.C., Lei, M., Zhong, Y., Hou, Y.J., et al. (2017). A pair of transposon-derived proteins function in a histone acetyltransferase complex for active DNA demethylation. Cell Res 27, 226-240. 
Emsley, P., Lohkamp, B., Scott, W.G., and Cowtan, K. (2010). Features and development of Coot. Acta Crystallogr D Biol Crystallogr 66, 486-501.

Furner, I.J., and Matzke, M. (2011). Methylation and demethylation of the Arabidopsis genome. Curr Opin Plant Biol 14, 137-141.

Gehring, M., Huh, J.H., Hsieh, T.F., Penterman, J., Choi, Y., Harada, J.J., Goldberg, R.B., and Fischer, R.L. (2006). DEMETER DNA glycosylase establishes MEDEA polycomb gene self-imprinting by allele-specific demethylation. Cell 124, 495-506.

Goldschmidt, L., Cooper, D.R., Derewenda, Z.S., and Eisenberg, D. (2007). Toward rational protein crystallization: A Web server for the design of crystallizable protein variants. Protein Sci 16, 1569-1576.

Gong, Z., Morales-Ruiz, T., Ariza, R.R., Roldan-Arjona, T., David, L., and Zhu, J.K. (2002). ROS1, a repressor of transcriptional gene silencing in Arabidopsis, encodes a DNA glycosylase/lyase. Cell 111, 803-814.

Harris, C.J., and Jacobsen, S.E. (2019). ADCP1: a novel plant H3K9me2 reader. Cell Res 29, 6-7.

Hsieh, T.F., Ibarra, C.A., Silva, P., Zemach, A., Eshed-Williams, L., Fischer, R.L., and Zilberman, D. (2009). Genome-wide demethylation of Arabidopsis endosperm. Science $324,1451-1454$.

Huh, J.H., Bauer, M.J., Hsieh, T.F., and Fischer, R.L. (2008). Cellular programming of plant gene imprinting. Cell 132, 735-744. 
Law, J.A., and Jacobsen, S.E. (2010). Establishing, maintaining and modifying DNA methylation patterns in plants and animals. Nat Rev Genet 11, 204-220.

Lang Z, Lei M, Wang X, Tang K, Miki D, Zhang H, Mangrauthia SK, Liu W, Nie W, Ma $\mathrm{G}$, et al. (2015). The methyl-CpG-binding protein MBD7 facilitates active DNA demethylation to limit DNA hyper-methylation and transcriptional gene silencing. Mol Cell 57, 971-983.

Lei, M., La, H., Lu, K., Wang, P., Miki, D., Ren, Z., Duan, C.G., Wang, X., Tang, K., Zeng, L., et al. (2014). Arabidopsis EDM2 promotes IBM1 distal polyadenylation and regulates genome DNA methylation patterns. Proc Natl Acad Sci U S A 111, 527-532.

Liu, R., and Lang, Z. (2020). The mechanism and function of active DNA demethylation in plants. J Integr Plant Biol 62, 148-159.

Lindroth AM, Cao X, Jackson JP, Zilberman D, McCallum CM, Henikoff S, Jacobsen SE. Requirement of CHROMOMETHYLASE3 for maintenance of CpXpG methylation. Science. 2001 Jun 15;292(5524):2077-80.

Marchler-Bauer, A., Bo, Y., Han, L., He, J., Lanczycki, C.J., Lu, S., Chitsaz, F., Derbyshire, M.K., Geer, R.C., Gonzales, N.R., et al. (2017). CDD/SPARCLE: functional classification of proteins via subfamily domain architectures. Nucleic Acids Res 45, D200-D203.

Maurer-Stroh, S., Dickens, N.J., Hughes-Davies, L., Kouzarides, T., Eisenhaber, F., and Ponting, C.P. (2003). The Tudor domain 'Royal Family': Tudor, plant Agenet, Chromo, PWWP and MBT domains. Trends Biochem Sci 28, 69-74. 
McBrian, M.A., Behbahan, I.S., Ferrari, R., Su, T., Huang, T.W., Li, K., Hong, C.S., Christofk, H.R., Vogelauer, M., Seligson, D.B., et al. (2013). Histone acetylation regulates intracellular pH. Mol Cell 49, 310-321.

Matzke MA, Mosher RA. RNA-directed DNA methylation: an epigenetic pathway of increasing complexity. Nat Rev Genet. 2014 Jun;15(6):394-408. doi: 10.1038/nrg3683. Epub 2014 May 8. Erratum in: Nat Rev Genet. 2014 Aug;15(8):570.

Matzke M, Kanno T, Daxinger L, Huettel B, Matzke AJ. RNA-mediated chromatin-based silencing in plants. Curr Opin Cell Biol. 2009 Jun;21(3):367-76.

Nie, W.F., Lei, M., Zhang, M., Tang, K., Huang, H., Zhang, C., Miki, D., Liu, P., Yang, Y., Wang, X., et al. (2019). Histone acetylation recruits the SWR1 complex to regulate active DNA demethylation in Arabidopsis. Proc Natl Acad Sci U S A 116, 16641-16650.

Otwinowski, Z., and Minor, W. (1997). Processing of X-ray diffraction data collected in oscillation mode. Methods Enzymol 276, 307-326.

Patel, D.J. (2016). A Structural Perspective on Readout of Epigenetic Histone and DNA Methylation Marks. Cold Spring Harb Perspect Biol 8, a018754.

Penterman, J., Zilberman, D., Huh, J.H., Ballinger, T., Henikoff, S., and Fischer, R.L. (2007). DNA demethylation in the Arabidopsis genome. P Natl Acad Sci U S A 104, 67526757.

Qian, W., Miki, D., Zhang, H., Liu, Y., Zhang, X., Tang, K., Kan, Y., La, H., Li, X., Li, S., et al. (2012). A histone acetyltransferase regulates active DNA demethylation in Arabidopsis. Science 336, 1445-1448. 
Qian W, Miki D, Lei M, Zhu X, Zhang H, Liu Y, Li Y, Lang Z, Wang J, Tang K, Liu R, Zhu JK. (2014). Regulation of active DNA demethylation by an $\alpha$-crystallin domain protein in Arabidopsis. Mol Cell 55, 361-71.

Ramos, A., Hollingworth, D., Adinolfi, S., Castets, M., Kelly, G., Frenkiel, T.A., Bardoni, B., and Pastore, A. (2006). The structure of the N-terminal domain of the fragile X mental retardation protein: a platform for protein-protein interaction. Structure 14, 21-31.

Robert, X., and Gouet, P. (2014). Deciphering key features in protein structures with the new ENDscript server. Nucleic Acids Res 42, W320-324.

Robertson, K.D. (2005). DNA methylation and human disease. Nat Rev Genet 6, 597-610.

Shen, J., Zeng, Y., Zhuang, X., Sun, L., Yao, X., Pimpl, P., and Jiang, L. (2013). Organelle $\mathrm{pH}$ in the Arabidopsis endomembrane system. Mol Plant 6, 1419-1437.

Sijacic P, Holder DH, Bajic M, Deal RB. Methyl-CpG-binding domain 9 (MBD9) is required for $\mathrm{H} 2 \mathrm{~A} . \mathrm{Z}$ incorporation into chromatin at a subset of $\mathrm{H} 2 \mathrm{~A} . \mathrm{Z}$-enriched regions in the Arabidopsis genome. PLoS Genet. 2019 Aug 5;15(8):e1008326.

Slotkin, R.K., and Martienssen, R. (2007). Transposable elements and the epigenetic regulation of the genome. Nat Rev Genet 8, 272-285.

Tencer, A.H., Gatchalian, J., Klein, B.J., Khan, A., Zhang, Y., Strahl, B.D., van Wely, K.H.M., and Kutateladze, T.G. (2017). A Unique pH-Dependent Recognition of Methylated Histone H3K4 by PPS and DIDO. Structure 25, 1530-1539 e1533. 
Wang, Y., Jin, J., Chung, M.W.H., Feng, L., Sun, H., and Hao, Q. (2018). Identification of the YEATS domain of GAS41 as a pH-dependent reader of histone succinylation. Proc Natl Acad Sci U S A 115, 2365-2370.

Zemach A, Kim MY, Hsieh PH, Coleman-Derr D, Eshed-Williams L, Thao K, Harmer SL, Zilberman D. The Arabidopsis nucleosome remodeler DDM1 allows DNA methyltransferases to access H1-containing heterochromatin. Cell. 2013 Mar 28;153(1):193-205.

Zhang, C., Du, X., Tang, K., Yang, Z., Pan, L., Zhu, P., Luo, J., Jiang, Y., Zhang, H., Wan, H., et al. (2018a). Arabidopsis AGDP1 links H3K9me2 to DNA methylation in heterochromatin. Nat Commun 9, 4547.

Zhang, H., Lang, Z., and Zhu, J.K. (2018b). Dynamics and function of DNA methylation in plants. Nat Rev Mol Cell Biol 19, 489-506.

Zhao, S., Cheng, L., Gao, Y., Zhang, B., Zheng, X., Wang, L., Li, P., Sun, Q., and Li, H. (2019). Plant HP1 protein ADCP1 links multivalent H3K9 methylation readout to heterochromatin formation. Cell Res 29, 54-66.

Zhu, J.K. (2009). Active DNA Demethylation Mediated by DNA Glycosylases. Annu Rev Genet 43, 143-166. 


\section{Figure Legends}

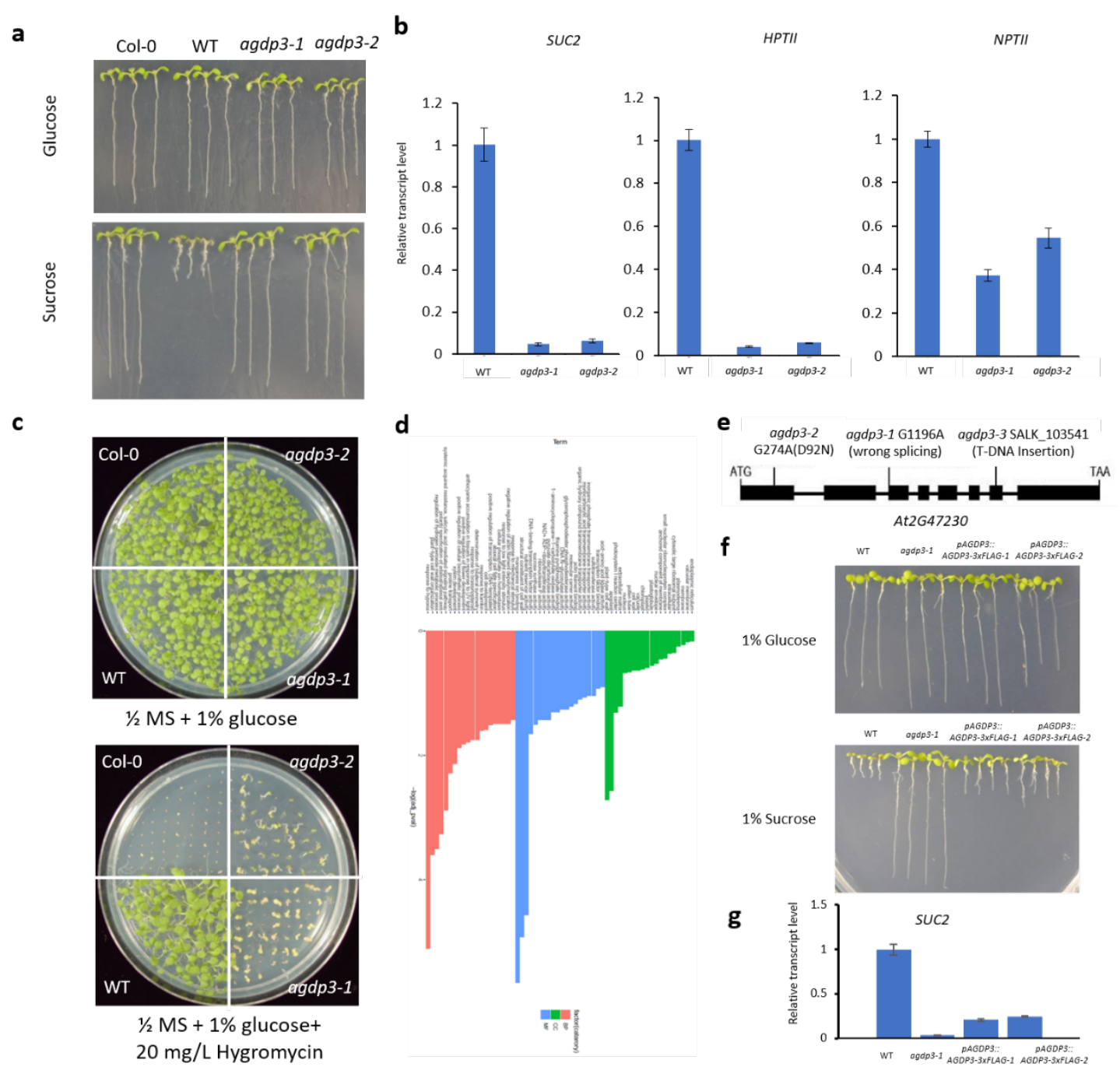

Figure. 1. AGDP3 Prevents DNA Hypermethylation and Transcriptional Gene

Silencing. a Comparison of root growth phenotype among Col-0, 35S::SUC transgenic line (WT), and $a g d p 3$ mutants grown on glucose or sucrose medium. $\mathbf{b}$ Transcript levels of SUC2, HPTII and NPTII transgenes in agdp3-1 and agdp3-2 mutants compared to WT detected by RT-qPCR. $\mathbf{c}$ AGDP3 dysfunction causes compromised hygromycin resistance in the transgenic plants. d RNA-Seq analysis of WT and agdp3-1 seedlings. e A diagram of the $A G D P 3$ gene showing the mutation in agdp3-1 and agdp3-2 mutant, and the T-DNA insertion position of agdp3-3 allele. Boxes and lines represent exons and introns, respectively. f Expression of $A G D P 3$ driven by its native promotor rescued the 
short root phenotype in agdp3-1. g SUC2 transcript level in WT, agdp3-1 and both complementation transgenic lines detected by RT-qPCR.

a

\begin{tabular}{|l|c|c|c|c|c|}
\hline Genotype & $\begin{array}{c}\text { Hyper- } \\
\text { DMR }\end{array}$ & $\begin{array}{c}\text { Hypo- } \\
\text { DMR }\end{array}$ & $\begin{array}{c}\text { overlap } \\
\text { with rdd }\end{array}$ & $\begin{array}{c}\text { overlap } \\
\text { with ros1-4 }\end{array}$ & $\begin{array}{c}\text { overlap with } \\
\text { idm1-1 }\end{array}$ \\
\hline rdd & 8276 & 1072 & & & \\
\hline ros1-4 & 5197 & 748 & $62.9 \%$ & & \\
\hline idm1-1 & 830 & 753 & $49.3 \%$ & $46.5 \%$ & \\
\hline agdp3-3 & 909 & 566 & $54.1 \%$ & $50.6 \%$ & $22.0 \%$ \\
\hline
\end{tabular}

b

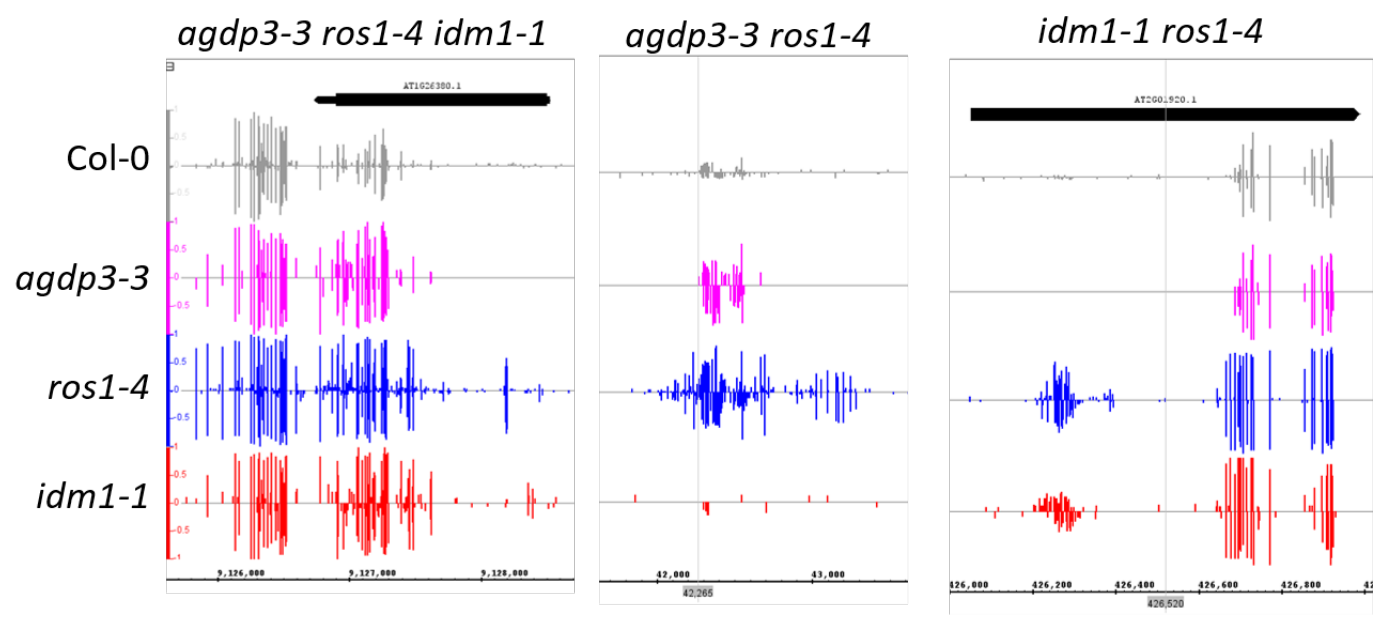

Figure. 2. Analysis of DMRs identified in agdp3-3 mutant. a The numbers of DMRs identified in $r d d$, ros 1-4, idm 1-1 and agdp3-3 and the overlap of hyper-DMRs between genotypes. b Examples of hyper-DMRs showing different pattern in ros 1-4, idm 1-1 and agdp3-3. 
a

Chromosomal Binding Peaks

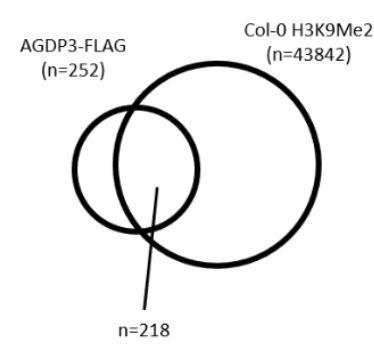

Overlapping Rate: $86.5 \%$ b

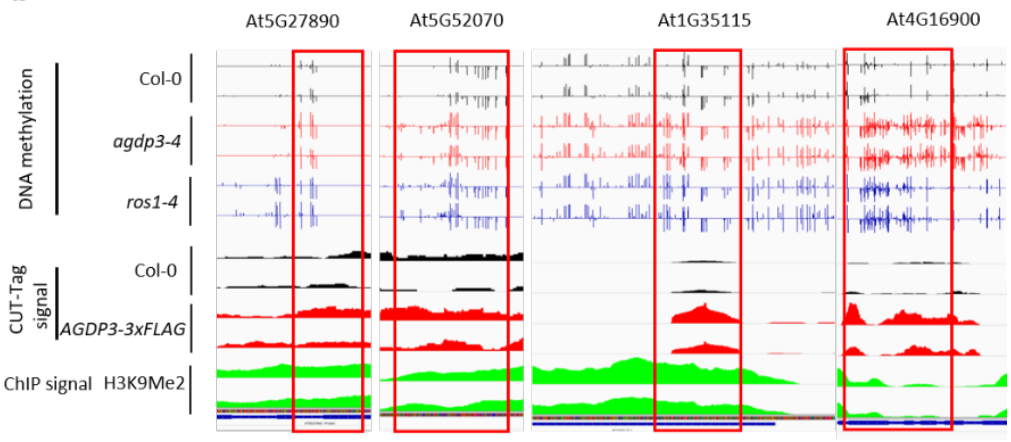

Figure. 3. a Overlap of binding peaks between AGDP3 and H3K9Me2. b Examples of DNA methylation, AGDP3 enrichment and H3K9Me2 signal at several selected common hyper-DMRs in ros 1-4 and agdp3-3.
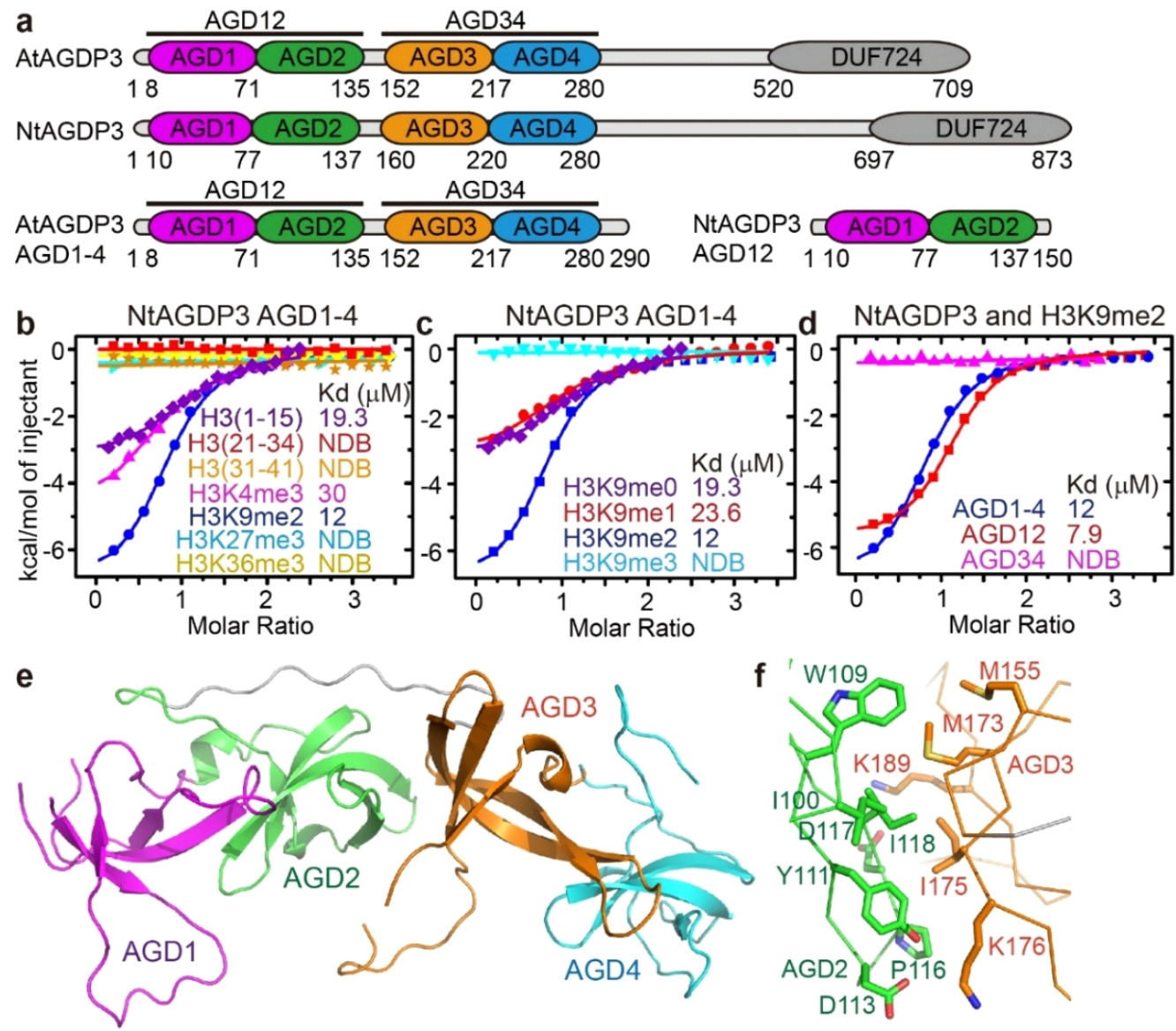

Fig. 4. Overall structure of AGDP3 AGD1-4. a The schematic representation of the domain architecture of AtAGDP3 (upper panel), NtAGDP3 (middle panel), and the 
constructs used for structural studies (lower panel). b-c The ITC binding curves between NtAGDP3 AGD1-4 and various unmodified and methylated histone peptides (b) and different methylated status of H3K9 (c) reveal AGDP3 is an H3K9me2-specific reader. NDB, no detectable binding. $\mathbf{d}$ The ITC binding curves between different AGDP3 AGD cassettes and $\mathrm{H} 3 \mathrm{~K} 9 \mathrm{me} 2$ reveal that the AGD12 cassette of AGDP3 is responsible for the H3K9me2 binding. e Overall structure of AtAGDP3 AGD1-4 with the four AGDs colored in magenta, green, orange, and cyan, respectively. $\mathbf{f}$ The interacting interface between AGD2 and AGD3 of AtAGDP3 with the interacting residues highlighted in stick models.
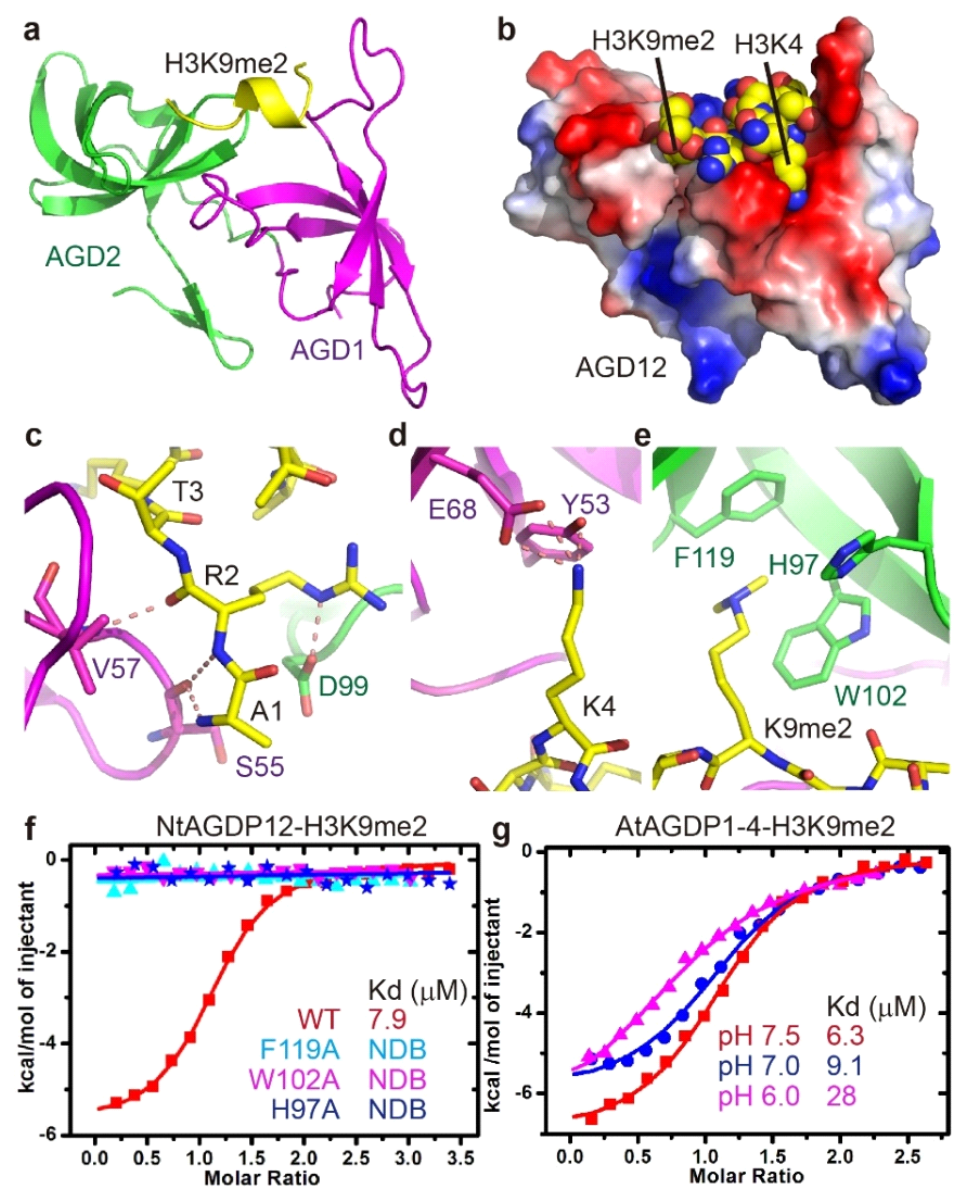

Fig. 5. Structure of NtAGDP3 AGD12 in complex with an H3K9me2 peptide. a A ribbon diagram of the overall structure of NtAGDP3 AGD12-H3K9me2 complex with the AGD1, AGD2, and the peptide colored magenta, green, and yellow, respectively. The 
H3K9me2 peptide adopts a helical conformation. b An electrostatic surface view of the NtAGDP3 AGD12 with the H3K9me2 peptide in space filling model. The unmodified H3K4 and dimethylated H3K9 insert their side chains into two adjacent surface pockets of AGD12. c-e The detailed interaction between AGD12 and the peptide residues H3A1 and H3R2 (c), H3K4 (d), and H3K9me2 (e). The interacting residues and hydrogen bonds are highlighted in stick and dashed red lines, respectively. $\mathbf{f}$ The ITC binding curves between different AGD12 mutations that disrupt the H3K9me2 binding aromatic cage and $\mathrm{H} 3 \mathrm{~K} 9 \mathrm{me} 2$ peptide revealing the aromatic cage is essential for the peptide binding. $\mathbf{g}$ The ITC binding curves between AtAGDP3 AGD1-4 and H3K9me2 peptide in different $\mathrm{pH}$ indicating AGDP3 is a $\mathrm{pH}$-dependent $\mathrm{H} 3 \mathrm{~K} 9 \mathrm{me} 2$ binding module. NDB, no detectable binding.

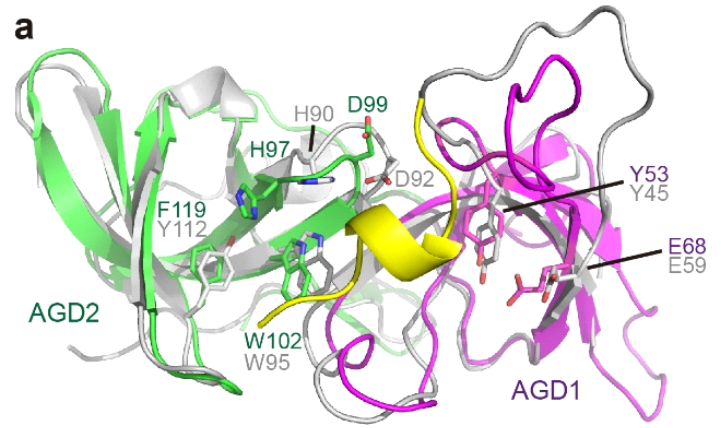

NtAGDP3 AGD1 AGD2 - H3K9me2 complex AtAGDP3 AGD1 AGD2

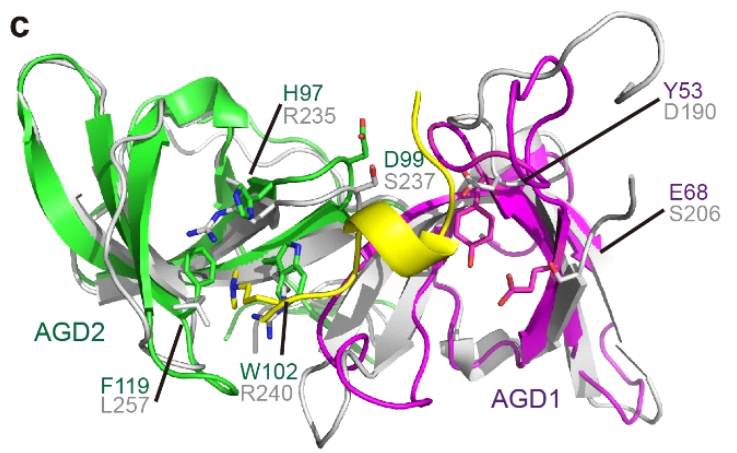

NtAGDP3 AGD1 AGD2 - H3K9me2 complex AtAGDP1 AGD3 AGD4

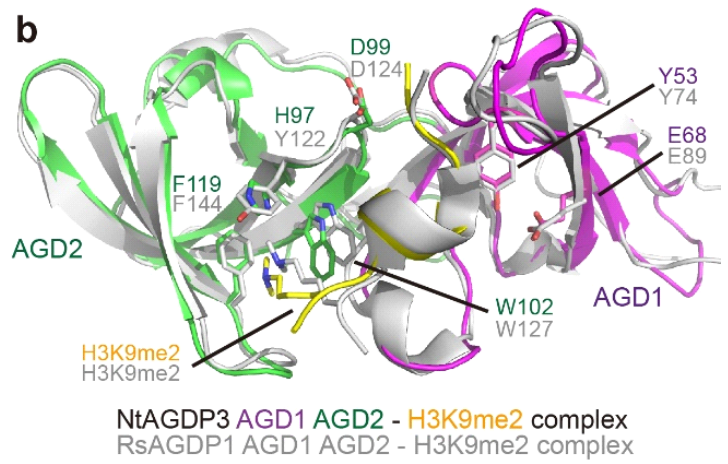

d AtAGDP1-4-H90Y-H3K9me2

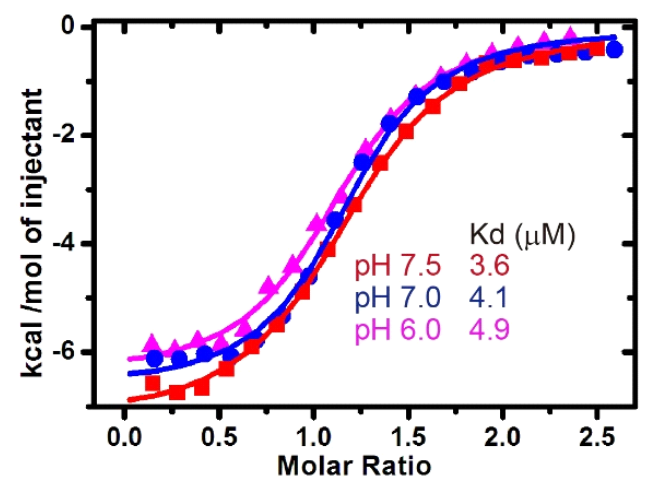

Fig. 6. The structural comparison of different tandem AGDs and AGDP3 is a pH dependent H3K9me2 reader. a The superimposition of NtAGDP3 AGD12-H3K9me2 with the free form AtAGDp3 AGD12 shows almost identical conformation and key residue positions, suggesting that AtAGDP3 AGD12 recognizes H3K9me2 in a similar 
way as NtAGDP3 AGD12. b The superimposition of NtAGDP3 AGD12-H3K9me2 complex and RsAGDP1 AGD12-H3K9me2 complex shows similar conformation and key residue positions, indicating similar $\mathrm{H} 3 \mathrm{~K} 9 \mathrm{me} 2$ recognition mechanism. The major difference is that the aromatic residues Tyr122 in RsAGDP1 is replaced by His97 in NtAGDP3. c The superimposition of NtAGDP3 AGD12 and AtAGDP3 AGD34 shows that the aromatic cage residues of AGD12 is not conserved in AGD34, suggesting AGD34 is not a methyl lysine reader and may possess other functions. $\mathbf{d}$ The ITC measurement of AtAGDP3 AGD1-4 H90Y mutant with H3K9me2 in different pH shows similar binding affinity, suggesting that the histidine residues is responsible for the $\mathrm{pH}$ dependence.

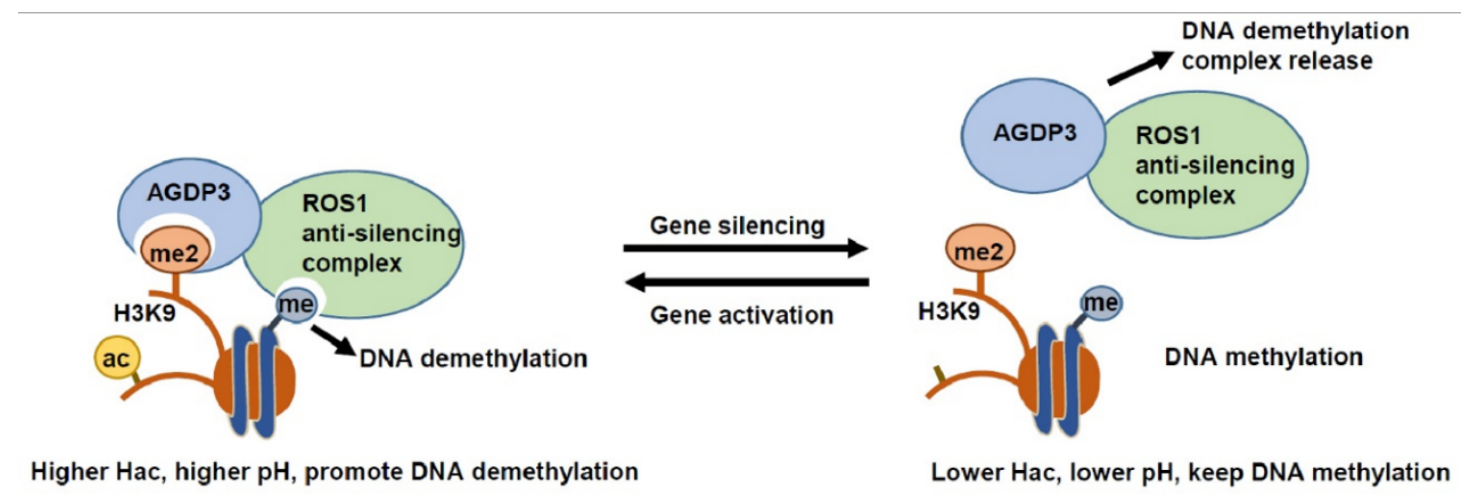

Fig. 7. A schematic model for the molecular function of AGDP3. In gene activation loci, the active mark histone acetylation-associated high $\mathrm{pH}$ may enable the binding of H3K9me2 by AGDP3, which further recruits ROS1 anti-silencing complex through an unknown factor to remove DNA methylation (left panel). In contrast, in gene silencing loci, the histone deacetylation associated low $\mathrm{pH}$ may be sensed by AGDP3 and further release the $\mathrm{H} 3 \mathrm{~K} 9 \mathrm{me} 2$ binding, allowing the ROS1 anti-silencing complex to be released and keep DNA methylation (right panel). 